\title{
Objective Attributes Weights Determining Based on Shannon Information Entropy in Hesitant Fuzzy Multiple Attribute Decision Making
}

\author{
Yingjun Zhang, ${ }^{1}$ Yizhi Wang, ${ }^{1}$ and Jingping Wang ${ }^{2}$ \\ ${ }^{1}$ Beijing Key Lab of Traffic Data Analysis and Mining, School of Computer and Information Technology, \\ Beijing Jiaotong University, Beijing 100044, China \\ ${ }^{2}$ School of Electronics and Information Engineering, Harbin Institute of Technology, Harbin 150001, China \\ Correspondence should be addressed to Yingjun Zhang; hitzyj@163.com
}

Received 21 December 2013; Revised 18 March 2014; Accepted 18 March 2014; Published 7 April 2014

Academic Editor: Hamid Reza Karimi

Copyright (C) 2014 Yingjun Zhang et al. This is an open access article distributed under the Creative Commons Attribution License, which permits unrestricted use, distribution, and reproduction in any medium, provided the original work is properly cited.

\begin{abstract}
Hesitant fuzzy set has been an important tool in dealing with multiple attribute decision making (MADM) problems, especially for the decision making situation when only some values of membership are possible for an alternative on attributes. However, determining attributes weights in hesitant fuzzy MADM is still an open problem. In this paper, we propose an objective weighting approach based on Shannon information entropy, which expresses the relative intensities of attribute importance to signify the average intrinsic information transmitted to the decision maker. Furthermore, we construct a hesitant fuzzy MADM approach based on the TOPSIS method and a weighted correlation coefficient proposed in this paper. Finally, we utilize a supplier selection example to validate the objective attributes weights determining method and the proposed hesitant fuzzy MADM approach.
\end{abstract}

\section{Introduction}

For a multiple attribute decision making (MADM) problem, an expert (decision maker) must evaluate every alternative to each attribute, assess attributes weights, and select the most desirable one from all the alternatives [1]. The MADM method provides a feasible and effective way to rank all the alternatives among noncommeasurable and conflicting attributes. Some representative approaches, such as the technique for order preference by similarity to ideal solution (TOPSIS) [2], the simple additive weighted (SAW) method [3], the ordered weighted averaging (OWA) method [4], and the analytic hierarchy process (AHP) [5], have been successfully utilized in dealing with MADM problems. However, in most MADM situations, the preference of attributes over alternatives provided by decision makers is usually not sufficient for the crisp numerical data, because things are fuzzy, uncertain, and probably influenced by the subjectivity of the decision makers, or the knowledge and data about the problem domain are insufficient during the decision making process [6]. To solve those MADM problems with uncertainty, research has extended the use of fuzzy set theory introduced by Zadeh to develop various MADM methods [7]. On the basis of Zadeh's fuzzy set, Torra and Narukawa $[8,9]$ introduced hesitant fuzzy set (HFS), which allows the membership of an element to a set derived from a few different values. HFS provides an effective way in dealing with decision making situation when only some values of membership are possible for an alternative on attributes. After the pioneering work of Torra and Narukawa, a fair proportion of literatures have done the theoretical and application research within the framework of HFS. Torra and Narukawa $[8,9]$ firstly generalized existing operations on fuzzy sets to HFS and discussed their application in decision making. $\mathrm{Xu}$ and $\mathrm{Xia}$ introduced distance, similarity, and correlation coefficient on HFS and utilized these operations to solve hesitant fuzzy MADM problems on the basis of TOPSIS method [10-12]. Xia and Xu [13] and Zhang [14] proposed various aggregation operators on HFS, which are effective in dealing with different decision making situations. 
Chen et al. [15] presented a clustering method for HFSs based on correlation coefficient. Yu et al. [16] extended Choquet integral on HFS and discussed its application in uncertain decision making situations. Zhu et al. [17] took into account the interrelationships among arguments and proposed hesitant fuzzy geometric Bonferroni means in dealing with hesitant fuzzy MADM problems. Wei [18] presented hesitant fuzzy prioritized operators for solving the decision making problems with different priority levels' attributes. On the basis of HFS and intervalued fuzzy set, generalized HFS and interval-valued HFS were proposed and utilized in decision making situations [19-21]. Rodriguez et al. [22] utilized hesitant fuzzy linguistic term set as an extension of HFS to solve uncertain qualitative decision making settings.

It is necessary to select appropriate attributes weights in decision making situations since the varied values of attributes weights may result in different ranking order of alternatives. Generally speaking, the attributes weights are divided into objective attributes and subjective attributes according to the ways of information acquisition [23]. The subjective attributes weights are obtained by preference information on the attributes given by the decision maker, who provides subjective intuition or judgments on specific attributes. AHP method [5] and Delphi method [24] are classical approaches for determining subjective attributes weights based on the preference of decision maker. Determining objective attributes weights depends on the decision making matrix. The existing objective attributes weights determining approaches include entropy-based method and optimization methods [25-27]. Most research pertaining to MADM analysis under hesitant fuzzy environment has been utilized depending on existing attributes weights [11-14, 18]. However, there is little research focusing on the problems of assessing objective attributes weights in hesitant fuzzy MADM.

In this paper, we present a new objective attributes weighting method based on Shannon information entropy in hesitant fuzzy MADM. The new objective attributes weighting method expresses the relative intensities of attribute importance to signify the average intrinsic information derived from decision maker, which has an emphasis on the discrimination among data to assess attributes weights. On the basis of known attributes weights, we construct a hesitant fuzzy MADM approach based on TOPSIS method and a weighted correlation coefficient proposed in this paper. The weighted correlation coefficient proposed in this paper takes into account the divergence among different elements on two HFSs. Therefore, the new correlation coefficient is helpful to reflect the attributes' importance in decision making situations.

The rest of this paper is organized as follows. Section 2 presents the concept of HFS and some of its basic operations. Section 3 presents a new weighted correlation coefficient within the framework of HFSs. Section 4 recalls Shannon information entropy and proposes a new objective attributes weighting method and a hesitant fuzzy MADM approach. Section 5 illustrates the proposed objective weighting method and the hesitant fuzzy MADM approach through a supplier selection example. Section 6 draws a conclusion.

\section{HFS and Its Operations}

In this section, we briefly recall HFS and some of its relevant operations.

\subsection{HFS}

Definition 1 (see $[8,9])$. Let $X$ be a reference set; a HFS on $X$ is defined in terms of a function that when applied to $X$ returns a subset of $[0,1]$, which can be represented by the following mathematical symbol:

$$
E=\left\{x, h_{E}(x) \mid x \in X\right\},
$$

where $h_{E}(x)$ is a set of values in $[0,1]$, denoting the possible membership degrees of the element $x \in X$ to the set $E$.

For convenience, we call $h_{E}(x)$ a hesitant fuzzy element (HFE).

\subsection{Operations on HFS}

Definition 2 (see [9]). Given a HFS $h_{E}(x)$, Torra defined its lower and upper bounds as follows:

$$
\begin{aligned}
& h_{E}^{-}(x)=\min h_{E}(x), \\
& h_{E}^{+}(x)=\max h_{E}(x),
\end{aligned}
$$

where $h_{E}^{-}(x)$ and $h_{E}^{+}(x)$ denote the lower and upper bound of $h_{E}(x)$, respectively.

Obviously, $\left(x, h_{E}^{-}(x), 1-h_{E}^{+}(x)\right)$ is an intuitionistic fuzzy set.

Definition 3 (see [8]). Given a HFS $h_{E}(x)$, the full set of HFS is as follows: $h_{E}(x)=\{1\}$ for all $x \in X$.

The definition of HFS implies that the number of values in different HFEs may be different. Xu and Xia [11] introduced $l\left(h_{E}(x)\right)$ to denote the number of values in $h_{E}(x)$. Assume the elements in $h_{E}(x)$ are in ascending order, and $h_{E}^{\sigma(j)}(x)$ is the $j$ th largest value in $h_{E}(x)$.

Definition 4 (see [13]). For a HFE $h_{E}(x)$, the score function $s\left(h_{E}(x)\right)$ is defined as follows:

$$
s\left(h_{E}(x)\right)=\frac{\sum_{j=1}^{l\left(h_{E}(x)\right)} h_{E}^{\sigma(j)}(x)}{l\left(h_{E}(x)\right)},
$$

where $s\left(h_{E}(x)\right) \in[0,1]$.

According to the score function on HFSs, $\mathrm{Xia}$ and $\mathrm{Xu}$ [13] introduced a method for ranking HFEs. For two HFEs $h_{1}$ and $h_{2}$, if $s\left(h_{1}\right)>s\left(h_{2}\right)$, then $h_{1}>h_{2}$; if $s\left(h_{1}\right)=s\left(h_{2}\right)$, then $h_{1}=h_{2}$.

\section{Correlation Coefficient on HFS}

Correlation coefficient on fuzzy set plays an important role in both theoretical and application fields, such as fuzzy pattern recognition, fuzzy clustering, artificial intelligence, and uncertain decision making. In some situations [11], 
the weights of each element $x_{i} \in X$ should be taken into account; we introduce a weighted correlation coefficient on HFS.

Definition 5. For two HFSs $A$ and $B$ on $X=\left\{x_{1}, x_{2}, \ldots, x_{n}\right\}$, a weighted correlation coefficient $C(A, B)$ is defined as

$$
\begin{gathered}
C(A, B)=\frac{1}{2}\left(C_{1}(A, B)+C_{2}(A, B)\right), \\
C_{1}(A, B)=1-\left[\sum_{i=1}^{n}\left|\frac{w_{i} \bar{h}_{A}\left(x_{i}\right)}{\left(\sum_{i=1}^{n}\left(w_{i} \bar{h}_{A}\left(x_{i}\right)\right)^{\lambda}\right)^{1 / \lambda}}\right|^{\lambda}\right]^{1 / \lambda} \\
C_{2}(A, B)=1-\left[\sum_{i=1}^{n} \mid \frac{\left(\sum_{i=1}^{n}\left(w_{i} \bar{h}_{B}\left(x_{i}\right)\right)^{\lambda}\right)^{1 / \lambda}}{\left(\sum_{i=1}^{n}\left(w_{i} \tilde{h}_{A}\left(x_{i}\right)\right)^{\lambda}\right)^{1 / \lambda}},\right. \\
-\left.\frac{w_{i} \tilde{h}_{A}\left(x_{i}\right)}{\left(\sum_{i=1}^{n}\left(w_{i} \tilde{h}_{B}\left(x_{i}\right)\right)^{\lambda}\right)^{1 / \lambda}}\right|^{\lambda}
\end{gathered}
$$

where $\bar{h}_{A}\left(x_{i}\right)=\sum_{j=1}^{l\left(h_{A}\left(x_{i}\right)\right)} h_{A}^{\sigma(j)}\left(x_{i}\right) / l\left(h_{A}\left(x_{i}\right)\right), \bar{h}_{B}\left(x_{i}\right)=$ $\sum_{j=1}^{l\left(h_{B}\left(x_{i}\right)\right)} h_{B}^{\sigma(j)}\left(x_{i}\right) / l\left(h_{B}\left(x_{i}\right)\right), \tilde{h}_{A}\left(x_{i}\right)=\sum_{j=1}^{l\left(h_{A}\left(x_{i}\right)\right)} h_{A}^{\sigma(j)}\left(x_{i}\right) /$ $\sum_{i=1}^{n} \sum_{j=1}^{l\left(h_{A}\left(x_{i}\right)\right)} h_{A}^{\sigma(j)}\left(x_{i}\right), \quad \tilde{h}_{B}\left(x_{i}\right)=\sum_{j=1}^{l\left(h_{B}\left(x_{i}\right)\right)} h_{B}^{\sigma(j)}\left(x_{i}\right) /$ $\sum_{i=1}^{n} \sum_{j=1}^{l\left(h_{B}\left(x_{i}\right)\right)} h_{B}^{\sigma(j)}\left(x_{i}\right), \lambda>0, w_{i} \in[0,1]$, and $\sum_{i=1}^{n} w_{i}=1$.

It is clear that $C(A, B)$ satisfies the following three properties of correlation coefficient on HFS:

(1) $|C(A, B)| \leq 1$,

(2) if $A=B$, then $C(A, B)=1$,

(3) $C(A, B)=C(B, A)$.

Proof. Obviously, $C_{1}(A, B) \leq 1$ and $C_{2}(A, B) \leq 1 ; C(A, B) \leq$ 1 holds. In the following part, we prove that $C(A, B) \geq-1$. Since

$$
\begin{gathered}
{\left[\sum_{i=1}^{n}\left|\frac{w_{i} \bar{h}_{A}\left(x_{i}\right)}{\left(\sum_{i=1}^{n}\left(w_{i} \bar{h}_{A}\left(x_{i}\right)\right)^{\lambda}\right)^{1 / \lambda}}-\frac{w_{i} \bar{h}_{B}\left(x_{i}\right)}{\left(\sum_{i=1}^{n}\left(w_{i} \bar{h}_{B}\left(x_{i}\right)\right)^{\lambda}\right)^{1 / \lambda}}\right|^{\lambda}\right]^{1 / \lambda}} \\
\quad \leq \sum_{i=1}^{n} \max \left\{\frac{\left(w_{i} \bar{h}_{A}\left(x_{i}\right)\right)^{\lambda}}{\sum_{i=1}^{n}\left(w_{i} \bar{h}_{A}\left(x_{i}\right)\right)^{\lambda}}, \frac{\left(w_{i} \bar{h}_{B}\left(x_{i}\right)\right)^{\lambda}}{\sum_{i=1}^{n}\left(w_{i} \bar{h}_{B}\left(x_{i}\right)\right)^{\lambda}}\right\} \leq 2,
\end{gathered}
$$

we get $C_{1}(A, B) \leq-1 .\left|C_{1}(A, B)\right| \leq 1$ holds. The proof of $\left|C_{2}(A, B)\right| \leq 1$ is the same as the proof process of $\left|C_{1}(A, B)\right| \leq$ 1. It implies that $|C(A, B)| \leq 1$ holds.

Obviously $C(A, B)$ satisfies properties (2).

The proof is completed.

\section{Hesitant Fuzzy MADM}

In this section, we propose an approach based on the TOPSIS method and the weighted correlation coefficient proposed in this paper to solve the hesitant fuzzy MADM problem with unknown attributes weights; particular emphasis is put on determining objective attributes weights based on Shannon information entropy.

4.1. Hesitant Fuzzy MADM Problem Description. A MADM problem can be regarded as a decision matrix whose elements denote the evaluation information of all alternatives in relation to an attribute. A hesitant fuzzy MADM problem is defined as below.

Assume that there are $m$ alternative measures, $A=$ $\left\{A_{1}, A_{2}, \ldots, A_{m}\right\}$, to be performed over $n$ attributes, $X=$ $\left\{x_{1}, x_{2}, \ldots, x_{n}\right\}$. The hesitant fuzzy decision matrix $D$ is expressed as follows:

$$
D=\left[\begin{array}{cccc}
h_{11} & h_{12} & \cdots & h_{1 n} \\
h_{21} & h_{22} & \cdots & h_{2 n} \\
\vdots & \vdots & \ddots & \vdots \\
h_{m 1} & h_{m 2} & \cdots & h_{m n}
\end{array}\right]
$$

where $h_{i j}=h_{A_{i}}\left(x_{j}\right)(i=1,2, \ldots, m ; j=1,2, \ldots, n)$ denotes a HFE.

4.2. Shannon Information Entropy. Shannon information entropy quantifies the expected value of the information contained in a message. Entropy is typically measured in bits, nats, or bans. Shannon information entropy is the average unpredictability in a random variable, which is equivalent to its information content.

Definition 6. Let $X=\left\{x_{1}, x_{2}, \ldots, x_{n}\right\}$ be a discrete random variable with probability mass function $P(X)$; the entropy $H$ of $X$ is defined as

$$
E(X)=D[-\ln (P(X))]
$$

where $D$ is the expected value operator. When taken from a finite sample, the entropy can explicitly be written as

$$
E(x)=-\sum_{i} P\left(x_{i}\right) \ln P\left(x_{i}\right)
$$

In relation to $P\left(x_{i}\right)=0$, the value of $0 \ln P(0)$ is taken to be 0 . 4.3. Objective Attributes Weighting Method. We first define a score matrix of a hesitant fuzzy decision making matrix. 
TABLE 1: Hesitant fuzzy decision matrix $D$.

\begin{tabular}{lcccc}
\hline & $X_{1}$ & $X_{2}$ & $X_{3}$ & $X_{4}$ \\
\hline$A_{1}$ & $\{0.5,0.4,0.3\}$ & $\{0.9,0.8,0.7,0.1\}$ & $\{0.5,0.4,0.2\}$ & $\{0.9,0.6,0.5,0.3\}$ \\
$A_{2}$ & $\{0.5,0.3\}$ & $\{0.9,0.7,0.6,0.5,0.2\}$ & $\{0.8,0.6,0.5,0.1\}$ & $\{0.7,0.4,0.3\}$ \\
$A_{3}$ & $\{0.7,0.6\}$ & $\{0.9,0.6\}$ & $\{0.7,0.5,0.3\}$ & $\{0.6,0.4\}$ \\
$A_{4}$ & $\{0.8,0.7,0.4,0.3\}$ & $\{0.7,0.4,0.2\}$ & $\{0.8,0.1\}$ & $\{0.9,0.8,0.6\}$ \\
$A_{5}$ & $\{0.9,0.7,0.6,0.3,0.1\}$ & $\{0.8,0.7,0.6,0.4\}$ & $\{0.9,0.8,0.7\}$ & $\{0.9,0.7,0.6,0.3\}$ \\
\hline
\end{tabular}

Definition 7 . Let $D$ be a hesitant fuzzy decision making matrix as (6). One calls

$$
S=\left[\begin{array}{cccc}
s_{11} & s_{12} & \cdots & s_{1 n} \\
s_{21} & s_{22} & \cdots & s_{2 n} \\
\vdots & \vdots & \ddots & \vdots \\
s_{m 1} & s_{m 2} & \cdots & s_{m n}
\end{array}\right]
$$

a score matrix of $D_{m \times n}$, where $s_{i j}=s\left(h_{i j}\right)$ is the score value of $h_{i j}$.

In the following part, we present a new objective attributes weighting method as follows.

Step 1. Calculate the score matrix $S$ of $D$.

Step 2. Normalize the score matrix $S$ as follows:

$$
\bar{S}=\left[\begin{array}{cccc}
\bar{s}_{11} & \bar{s}_{12} & \cdots & \bar{s}_{1 n} \\
\bar{s}_{21} & \bar{s}_{22} & \cdots & \bar{s}_{2 n} \\
\vdots & \vdots & \ddots & \vdots \\
\bar{s}_{m 1} & \bar{s}_{m 2} & \cdots & \bar{s}_{m n}
\end{array}\right],
$$

where $\bar{s}_{i j}=s_{i j} / \sum_{i=1}^{m} s_{i j}(i=1, \ldots, m ; j=1, \ldots, n)$.

Step 3. Determine the attributes weights.

Let

$$
E_{j}=-\frac{1}{\ln m} \sum_{i=1}^{m} \bar{s}_{i j} \ln \bar{s}_{i j}, \quad j=1, \ldots, n .
$$

The attribute weight $w_{j}(j=1, \ldots, n)$ is defined by

$$
w_{j}=\frac{1-E_{j}}{\sum_{j=1}^{n}\left(1-E_{j}\right)} .
$$

The above objective attributes weighting method utilizes Shannon information entropy to express the relative intensities of attribute importance and the divergence among attributes. And, then, the attributes weights are determined through (12).

4.4. Hesitant Fuzzy MADM Approach. With respect to the hesitant fuzzy MADM problem in Section 4.1, we present a hesitant fuzzy MADM approach based on TOPSIS method and the weighted correlation coefficient on HFS defined in Section 3. The schematic structure of the proposed hesitant fuzzy MADM approach is shown in Figure 1, and the detailed decision steps of this approach are listed as below.

Step 1. On the basis of Definitions 2 and 3, we define the hesitant fuzzy positive solution $A^{+}=\left\{\sigma_{1}^{+}, \sigma_{2}^{+}, \ldots, \sigma_{n}^{+}\right\}$, where $\sigma_{j}^{+}=\{1\}(j=1,2, \ldots, n)$.

Step 2. Determine the objective attributes weights $w=\left[w_{1}\right.$, $\left.w_{2}, \ldots, w_{n}\right]$ based on the newly objective weighting method in Section 4.3.

Step 3. Calculate the weighted correlation coefficient $C\left(A_{i}\right.$, $\left.A^{+}\right)(i=1,2, \ldots, m)$ using $(4)$.

Step 4. Rank all the alternatives $A_{i}$ based on $C\left(A_{i}, A^{+}\right)(i=$ $1,2, \ldots, m)$ and select the most desirable one.

\section{Illustrative Example and Discussion}

In this section, we utilize a supplier selection example to illustrate the proposed method for determining objective attributes weights in a MADM problem under hesitant fuzzy environment.

5.1. Illustrative Example. Suppose the supplier selection problem refers to 5 possible alternatives $A=\left\{A_{1}, A_{2}, A_{3}\right.$, $\left.A_{4}, A_{5}\right\}$ on 4 attributes $X=\left\{x_{1}, x_{2}, x_{3}, x_{4}\right\}$. The attributes weights for these problems are completely unknown. The hesitant fuzzy decision making matrix $D$ of $A$ on $X$ is shown in Table 1.

Step 1. Using Definitions 2 and 3, the hesitant fuzzy positive solution is defined as

$$
A^{+}=\{\{1\},\{1\},\{1\},\{1\}\}
$$

Step 2. Based on the new objective attributes weighting method, the process of determining attributes weights is as follows.

Firstly, calculate the score matrix of $D$ based on Definition 7:

$$
S=\left[\begin{array}{llll}
0.4000 & 0.6250 & 0.3667 & 0.5750 \\
0.4000 & 0.5800 & 0.5000 & 0.4667 \\
0.6500 & 0.7500 & 0.5000 & 0.5000 \\
0.5500 & 0.4333 & 0.4500 & 0.7667 \\
0.5200 & 0.6250 & 0.8000 & 0.6250
\end{array}\right]
$$


TABLE 2: The correlation coefficients and ranking results of all the alternatives.

\begin{tabular}{lcccccc}
\hline & $C\left(A_{1}, A\right)$ & $C\left(A_{2}, A\right)$ & $C\left(A_{3}, A\right)$ & $C\left(A_{4}, A\right)$ & $C\left(A_{5}, A\right)$ & Ranking results \\
\hline$\left.C\right|_{\lambda=1}$ & 0.7050 & 0.7981 & 0.8599 & 0.7100 & 0.9093 & $A_{5}>A_{3}>A_{2}>A_{4}>A_{1}$ \\
$\left.C\right|_{\lambda=2}$ & 0.7095 & 0.8052 & 0.8633 & 0.6885 & 0.9095 & $A_{5}>A_{3}>A_{2}>A_{1}>A_{4}$ \\
\hline
\end{tabular}

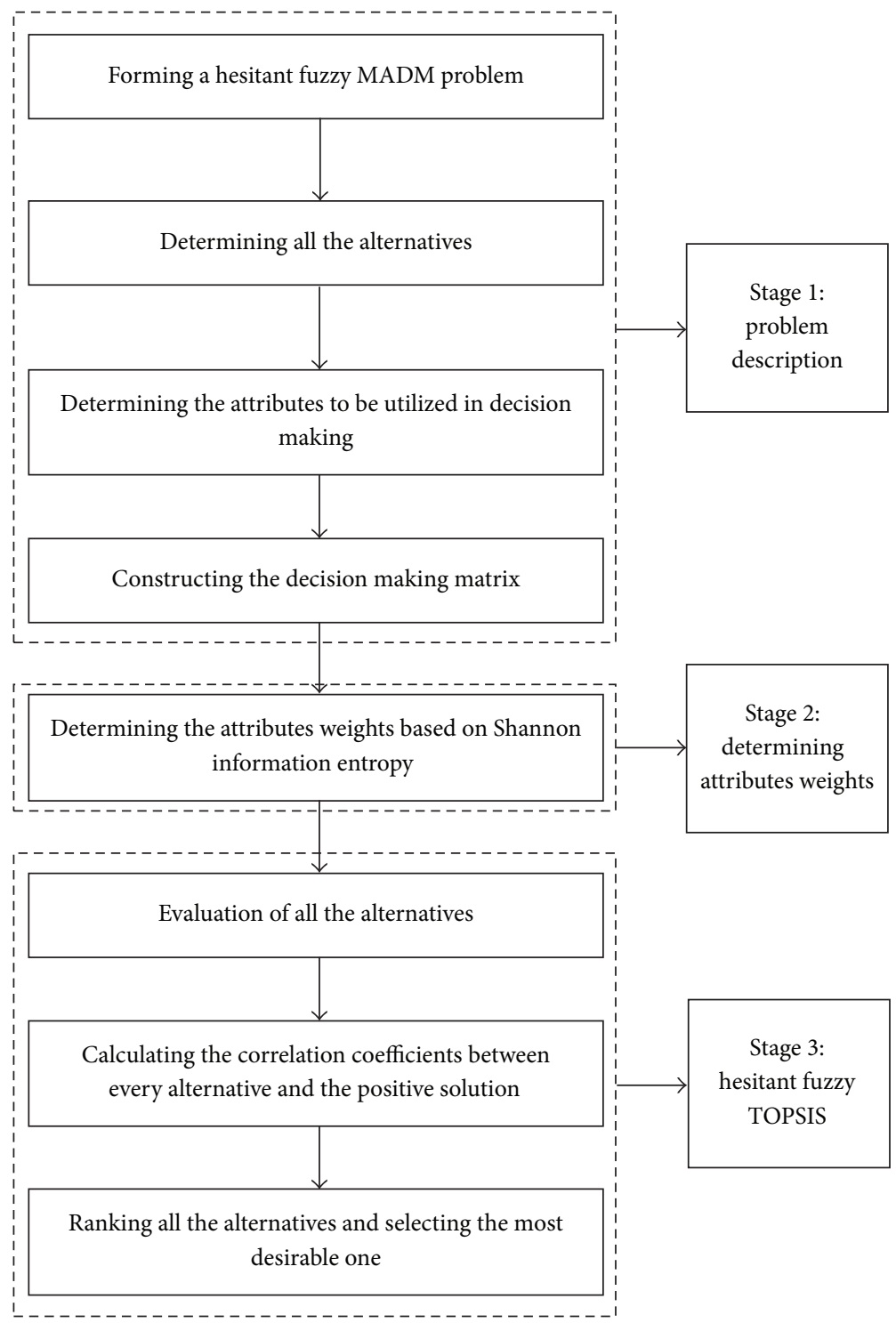

FIGURE 1: The schematic structure of the proposed hesitant fuzzy MADM approach.

Secondly, we get the the normalized score matrix $\bar{S}$ based on (10):

$$
\bar{S}=\left[\begin{array}{llll}
0.1587 & 0.2074 & 0.1401 & 0.1960 \\
0.1587 & 0.1925 & 0.1911 & 0.1591 \\
0.2579 & 0.2489 & 0.1911 & 0.1705 \\
0.2183 & 0.1438 & 0.1720 & 0.2614 \\
0.2063 & 0.2074 & 0.3057 & 0.2131
\end{array}\right]
$$

Thirdly, the attribute weight vector is determined by (11) and (12):

$$
w=\left[\begin{array}{llll}
0.2094 & 0.1735 & 0.4304 & 0.1867
\end{array}\right]^{T} .
$$

Step 3. Calculate the correlation coefficient $C\left(A_{i}, A^{+}\right)(i=$ $1,2,3,4)$ based on $C(\lambda=1,2)(4)$, and the results are shown as in Table 2. 
TABLE 3: The cross-entropy and ranking order of all the alternatives.

\begin{tabular}{ccccccc}
\hline & $A_{1}$ & $A_{2}$ & $A_{3}$ & $A_{4}$ & $A_{5}$ & Ranking results \\
\hline$C_{r}^{+}$ & 0.3080 & 0.2988 & 0.1990 & 0.2949 & 0.1583 & $A_{5}>A_{3}>A_{4}>A_{2}>A_{1}$ \\
$C_{r}^{-}$ & 0.3233 & 0.3083 & 0.3936 & 0.3704 & 0.4826 & $A_{5}>A_{3}>A_{4}>A_{1}>A_{2}$ \\
$C_{r}$ & 0.4879 & 0.4921 & 0.3358 & 0.4433 & 0.2470 & $A_{5}>A_{3}>A_{4}>A_{1}>A_{2}$ \\
\hline
\end{tabular}

Step 4. Rank all the alternatives based on the obtained correlation coefficient values, and the ranking results are shown as in Table 2.

5.2. Comparison with Existing Methods. In [12], Xu and Xia proposed a method based on hesitant fuzzy entropy and cross-entropy (Method $\mathrm{Xu}$ ) to deal with the hesitant fuzzy MADM problem with completely unknown attribute weight information. In the following part, we utilize Method $X u$ in an example in Section 5.1.

Step 1. Determine the attributes weights based on the weighting method of Method $\mathrm{Xu}$.

Firstly, calculate the hesitant fuzzy entropy matrix according to (44) with $q=2$ in [12]; we get the entropy matrix $E$ of D:

$$
E=\left[\begin{array}{llll}
0.9615 & 0.8790 & 0.9283 & 0.9742 \\
0.9613 & 0.9725 & 0.9898 & 0.9872 \\
0.9135 & 0.7527 & 1.0000 & 1.0000 \\
0.9900 & 0.9804 & 0.9891 & 0.7187 \\
0.9923 & 0.9368 & 0.6481 & 0.9357
\end{array}\right]
$$
[12]:

Secondly, calculate the attributes weights based on (53) in

$$
w=\left[\begin{array}{llll}
0.1218 & 0.3214 & 0.2987 & 0.2580
\end{array}\right]^{T} .
$$

Step 2. Based on the hesitant fuzzy cross-entropy (see (43) with $q=2$ in [12]), calculate the positive cross-entropy $C_{r}^{+}\left(A_{i}\right)(i=1, \ldots, 5)$, the negative cross-entropy $C_{r}^{-}\left(A_{i}\right)(i=$ $1, \ldots, 5)$, and the closeness degree $C_{r}\left(A_{i}\right)(i=1, \ldots, 5)$ of the alternative $A_{i}(i=1, \ldots, 5)$ to the ideal solution by (54)-(56) in [12] as in Table 3.

Step 3. Rank all the alternatives based on $C_{r}\left(A_{i}\right)(i=1, \ldots, 5)$ as in Table 3.

5.3. Discussion. Both Method $X u$ and the newly hesitant fuzzy MADM approach proposed in this paper choose $A_{5}$ as the most desirable one for the decision making example in Section 5.1. The results show that proposed MADM approach is effective for addressing hesitant fuzzy MADM problem with completely unknown attribute weight information. Instead of the decision making method in [12], which utilizes hesitant fuzzy entropy to determine attributes weights depending on the credibility of the input data, we employ Shannon information entropy to express the relative intensities of attribute importance and determine the objective attributes weights.

\section{Conclusion}

By applying hesitant fuzzy sets to uncertain MADM problems, we can get more accurate choice from the incomplete and complex information derived from decision makers in real life. In this paper, we propose a new objective attributes weighting method based on Shannon information entropy to express the relative intensities of attribute importance and determine the objective attributes weights. Furthermore, we propose a MADM approach based on the new objective weighting method and a weighted correlation coefficient introduced in this paper. The results of the example in Section 5.1 indicate that the newly MADM approach is feasible and effective in dealing with hesitant fuzzy MADM problems with completely unknown attributes weights. The newly hesitant fuzzy MADM approach offers a useful way to solve uncertain decision making problems derived from supplier selection, public risk, medical diagnosis, and other aspects.

\section{Conflict of Interests}

The authors declare that there is no conflict of interests regarding the publication of this paper.

\section{Acknowledgments}

The authors are very grateful to Professor Hamid Reza Karimi and two anonymous referees for their constructive comments and suggestions that have led to an improved version of this paper. This research was supported by Project funded by China Postdoctoral Science Foundation under Grant no. 2013M540848 and the Fundamental Research Funds for the Central Universities under Grant no. 2013JBM023.

\section{References}

[1] W. Ho, X. Xu, and P. K. Dey, "Multi-criteria decision making approaches for supplier evaluation and selection: a literature review," European Journal of Operational Research, vol. 202, no. 1, pp. 16-24, 2010.

[2] M. Behzadian, S. K. Otaghsara, M. Yazdani, and J. Ignatius, "A state-of the-art survey of TOPSIS applications," Expert Systems with Applications, vol. 39, no. 17, pp. 13051-13069, 2012.

[3] Y. S. Huang, W. C. Chang, W. H. Li, and Z. L. Lin, "Aggregation of utility-based individual preferences for group decisionmaking," European Journal of Operational Research, vol. 229, no. 2, pp. 462-469, 2013.

[4] R. R. Yager, "OWA aggregation over a continuous interval argument with applications to decision making," IEEE Transactions 
on Systems, Man, and Cybernetics B: Cybernetics, vol. 34, no. 5, pp. 1952-1963, 2004.

[5] R. J. Ormerod and W. Ulrich, "Operational research and ethics: a literature review," European Journal of Operational Research, vol. 228, no. 2, pp. 291-307, 2013.

[6] I. N. Durbach and T. J. Stewart, "Modeling uncertainty in multicriteria decision analysis," European Journal of Operational Research, vol. 223, no. 1, pp. 1-14, 2012.

[7] R. A. Ribeiro, "Fuzzy multiple attribute decision making: a review and new preference elicitation techniques," Fuzzy Sets and Systems, vol. 78, no. 2, pp. 155-181, 1996.

[8] V. Torra and Y. Narukawa, "On hesitant fuzzy sets and decision," in Proceedings of the IEEE International Conference on Fuzzy Systems, pp. 1378-1382, Jeju Island, Republic of Korea, August 2009.

[9] V. Torra, "Hesitant fuzzy sets," International Journal of Intelligent Systems, vol. 25, no. 6, pp. 529-539, 2010.

[10] Z. Xu and M. Xia, "On distance and correlation measures of hesitant fuzzy information," International Journal of Intelligent Systems, vol. 26, no. 5, pp. 410-425, 2011.

[11] Z. Xu and M. Xia, "Distance and similarity measures for hesitant fuzzy sets," Information Sciences, vol. 181, no. 11, pp. 2128-2138, 2011.

[12] Z. Xu and M. Xia, "Hesitant fuzzy entropy and cross-entropy and their use in multiattribute decision-making," International Journal of Intelligent Systems, vol. 27, pp. 799-822, 2012.

[13] M. Xia and Z. Xu, "Hesitant fuzzy information aggregation in decision making," International Journal of Approximate Reasoning, vol. 52, no. 3, pp. 395-407, 2011.

[14] Z. Zhang, "Hesitant fuzzy power aggregation operators and their application to multiple attribute group decision making," Information Sciences, vol. 234, pp. 150-181, 2013.

[15] N. Chen, Z. Xu, and M. Xia, "Correlation coefficients of hesitant fuzzy sets and their applications to clustering analysis," Applied Mathematical Modelling, vol. 37, no. 4, pp. 2197-2211, 2013.

[16] D. Yu, W. Zhang, and Y. Xu, "Group decision making under hesitant fuzzy environment with application to personnel evaluation," Knowledge-Based Systems, vol. 52, pp. 1-10, 2013.

[17] B. Zhu, Z. Xu, and M. Xia, "Hesitant fuzzy geometric Bonferroni means," Information Sciences, vol. 205, pp. 72-85, 2012.

[18] G. Wei, "Hesitant fuzzy prioritized operators and their application to multiple attribute decision making," Knowledge-Based Systems, vol. 31, pp. 176-182, 2012.

[19] B. Farhadinia, "Information measures for hesitant fuzzy sets and interval-valued hesitant fuzzy sets," Information Sciences, vol. 240, pp. 129-144, 2013.

[20] G. Qian, H. Wang, and X. Feng, "Generalized hesitant fuzzy sets and their application in decision support system," KnowledgeBased Systems, vol. 37, pp. 357-365, 2013.

[21] G. Wei, X. Zhao, and R. Lin, "Some hesitant interval-valued fuzzy aggregation operators and their applications to multiple attribute decision making," Knowledge-Based Systems, vol. 46, pp. 43-53, 2013.

[22] R. M. Rodriguez, L. Martinez, and F. Herrera, "Hesitant fuzzy linguistic term sets for decision making," IEEE Transactions on Fuzzy Systems, vol. 20, no. 1, pp. 109-119, 2012.

[23] E. K. Zavadskas, Z. Turskis, L. Ustinovichius, and G. Shevchenko, "Attributes weights determining peculiarities in multiple attribute decision making methods," Engineering Economics, no. 1, pp. 32-43, 2010.
[24] C. L. Hwang and M. J. Lin, Group Decision Making Under Multiple Criteria: Methods and Applications, Springer, Berlin, Germany, 1987.

[25] T. Y. Chen and C. H. Li, "Determining objective weights with intuitionistic fuzzy entropy measures: a comparative analysis," Information Sciences, vol. 180, no. 21, pp. 4207-4222, 2010.

[26] D. F. Li, “TOPSIS-based nonlinear-programming methodology for multiattribute decision making with interval-valued intuitionistic fuzzy sets," IEEE Transactions on Fuzzy Systems, vol. 18, no. 2, pp. 299-311, 2010.

[27] Y. Zhang, P. Li, Y. Wang, P. Ma, and X. Su, "Multiattribute decision making based on entropy under interval-valued intuitionistic fuzzy environment," Mathematical Problems in Engineering, vol. 2013, Article ID 526871, 8 pages, 2013. 


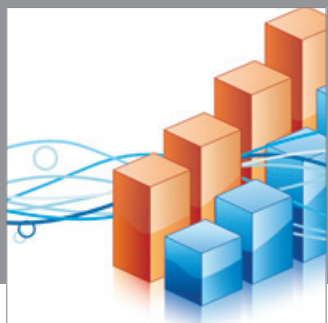

Advances in

Operations Research

mansans

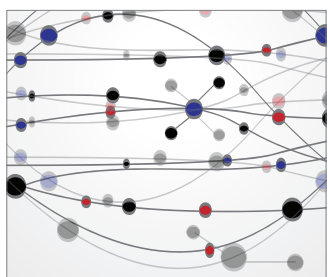

The Scientific World Journal
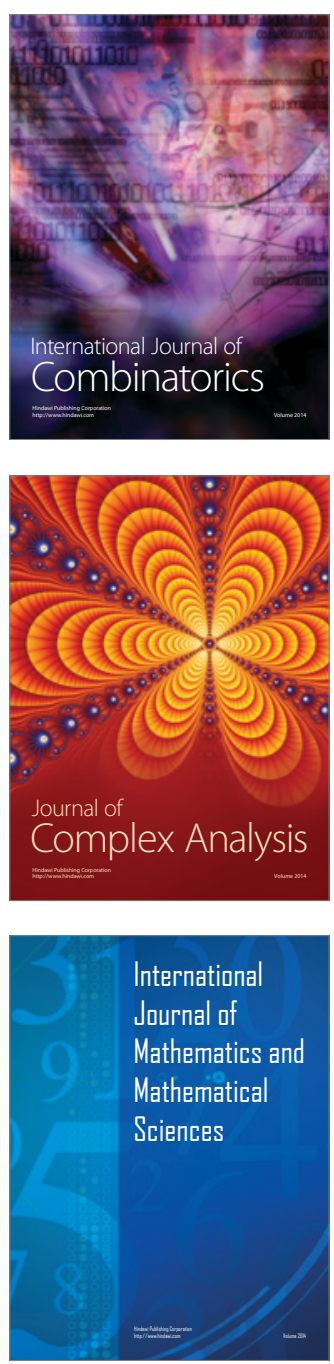
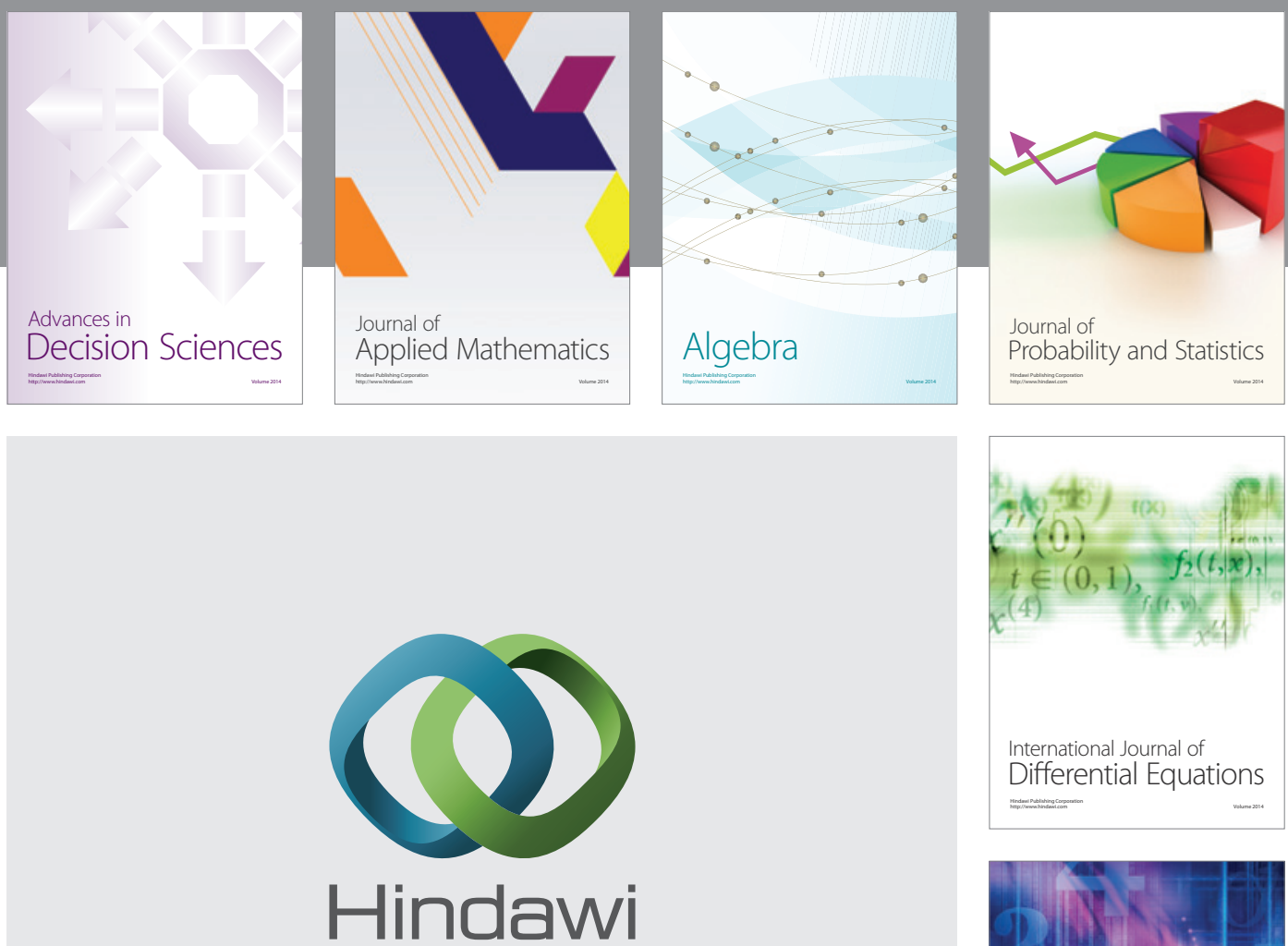

Submit your manuscripts at http://www.hindawi.com
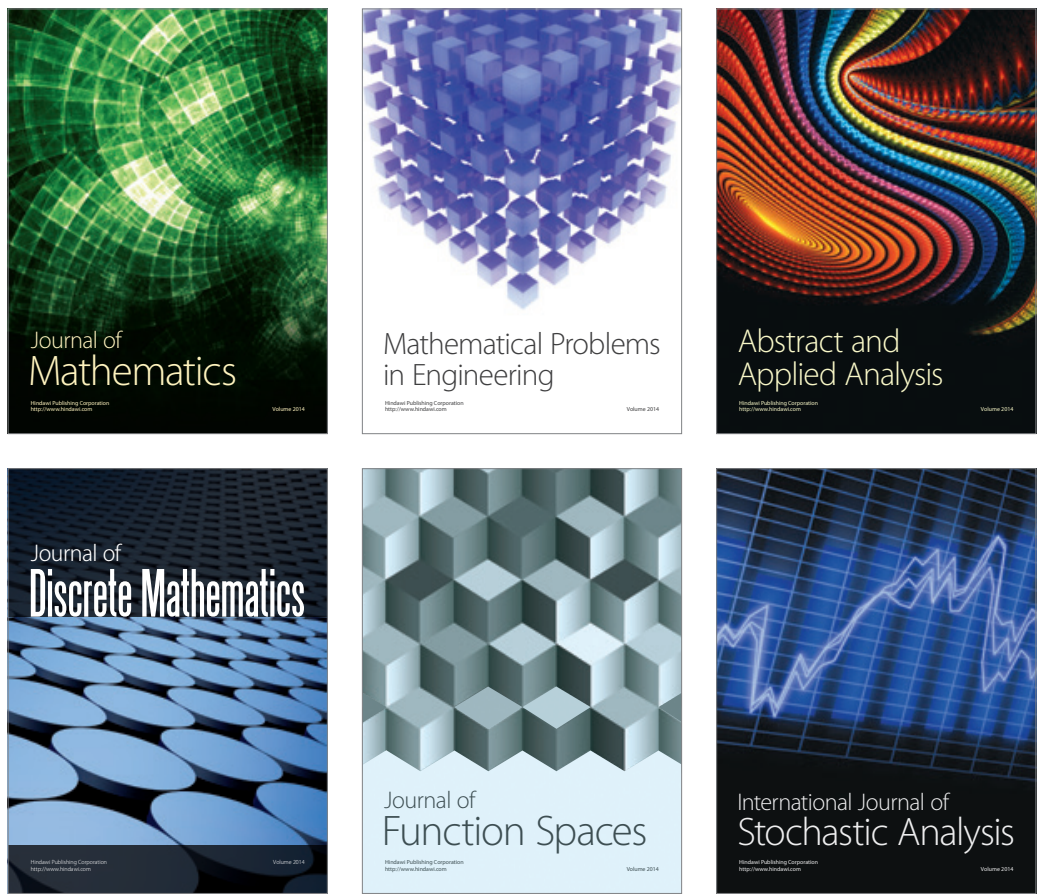

Journal of

Function Spaces

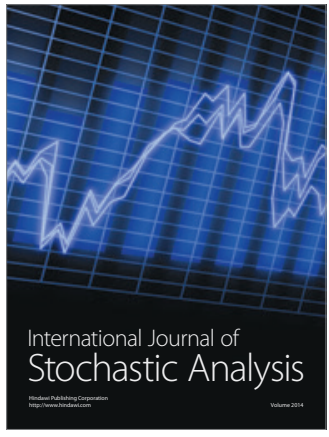

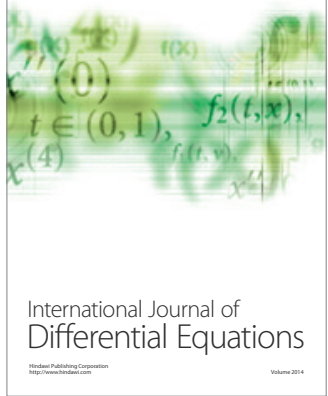
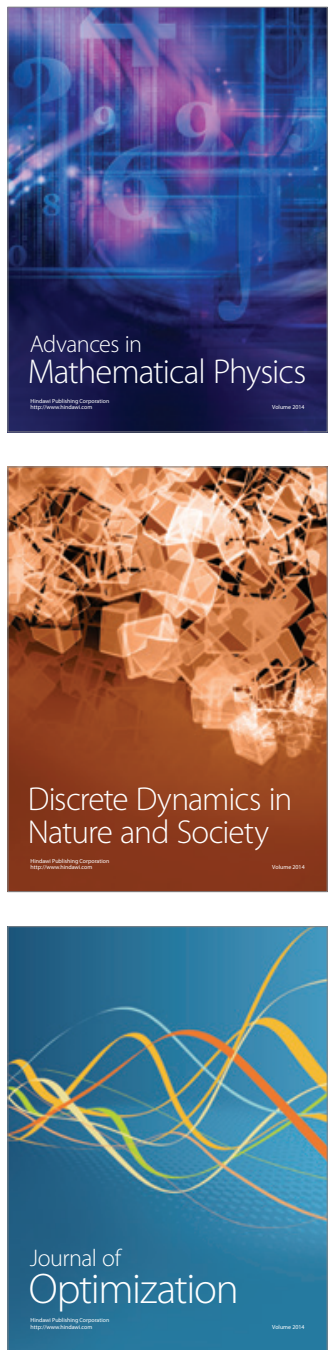